\title{
A THUE EQUATION WITH QUADRATIC INTEGERS AS VARIABLES
}

\author{
B. M. M. DE WEGER
}

\begin{abstract}
In an earlier paper we determined all the solutions in $\mathbb{Z}$ of a cubic Thue equation with coefficients in a quadratic number field. It is now shown that the method used there can be used to solve the more general problem of determining all the solutions of the Thue equation in a ring of quadratic integers.
\end{abstract}

\section{INTRODUCTION}

In the paper [4], we were interested in a cubic Thue equation with coefficients in a quadratic number field, but with rational integers as variables, namely

$$
x^{3}+(9+2 \sqrt{13}) x^{2} y-(12+\sqrt{13}) x y^{2}-\frac{11+3 \sqrt{13}}{2} y^{3}=\left(\frac{3+\sqrt{13}}{2}\right)^{n}
$$

(see [4, equation (18)]; we have replaced $2 n$ by $n$, to be slightly more general). We showed in [4, Theorem 3] that it has only the solutions $(x, y, n)=$ $(1,0,0),(0,-1,2)$.

It was only after the paper [4] was accepted for publication, that I realized that for the solution method used there it is not at all essential that $x, y$ be rational integers, and that the method in fact detects all the solutions $x, y$ in the ring of integers of $\mathbb{Q}(\sqrt{13})$, as will be shown below. We give our new results in $\S 2$, and details of the proof in $\S 3$. This will require only minor modifications in the proof of $[4, \S 3.3]$. As far as I know, this is the first time a Thue equation over a ring of quadratic integers is completely solved by Baker theory and computational diophantine approximation methods. Clearly, any "reasonable" Thue equation over a ring of integers can be treated by the same method.

All our results are based on a Key Lemma, which is about a unit equation in a sextic number field. This Key Lemma is given in $\S 3.1$, and was implicitly also present in [4], but in the proof presented there we used that $x, y \in \mathbb{Z}$. Now we remove this assumption from the proof. Moreover, we will use the recent very sharp result [1] by A. Baker and G. Wüstholz on linear forms in logarithms, to derive a new upper bound for the parameter $B$, replacing [4, equation (36)]. The new upper bound is only slightly larger than the (incorrect) one given in [4, equation (36)], and is equal to the corrected bound given in the Correction to [4], even though it holds in the present more general situation. Details are given below in $\S 3.2$.

Received by the editor February 2, 1994 and, in revised form, April 13, 1994.

1991 Mathematics Subject Classification. Primary 11D25, 11D41. 
Fortunately, it turns out that we do not have to redo the computational part of the first reduction step, as done in $[4, \S 3.4]$. However, the conclusion drawn there from these computations is again based on the assumption $x, y \in \mathbb{Z}$, so we have to redo the subsequent reduction steps. In $\S 3.3$ below we give details.

Finally, $\S 3.4$ contains the derivation of the results of $\S 2$ from the Key Lemma.

\section{A CUBIC THUe EQUATION OVER A QUADRATIC NUMBER FIELD AND RELATED EQUATIONS OF DEGREE SIX}

Let $\mathscr{O}$ denote the ring of integers $\mathscr{O}(\sqrt{13})$, and put $\alpha=\frac{3+\sqrt{13}}{2}$. Our main result is the following.

Theorem 1. The only solutions of equation (1) in $x, y \in \mathscr{O}$ and $n \in \mathbb{Z}$ are $(x, y, n)=\left(\alpha^{m}, 0,3 m\right),\left(0,-\alpha^{m}, 3 m+2\right)$, where $m$ runs through $\mathbb{Z}$.

In $\S 3.1$ we present the Key Lemma needed in the proof of this theorem. In $\S 3.4$ we show how this lemma implies Theorem 1 and some other results as by-products. As examples of such by-products we mention the following results on sextic diophantine equations with variables in $\mathbb{Z}$. Compare this with $[4$, equation (20)].

Theorem 2. (i) The diophantine equation

$$
v^{6}+18 v^{5} w+5 v^{4} w^{2}-175 v^{3} w^{3}+110 v^{2} w^{4}+93 v w^{5}+w^{6}=13^{3}
$$

in $v, w \in \mathbb{Z}$ has only the solutions $(v, w)= \pm(3,-1), \pm(4,3)$.

(ii) The diophantine equation

$$
t^{6}+4 t^{5} u-2 t^{4} u^{2}-9 t^{3} u^{3}+2 t^{2} u^{4}+4 t u^{5}-u^{6}= \pm 1
$$

in $t, u \in \mathbb{Z}$ has only the solutions $(t, u)= \pm(1,0), \pm(0,1), \pm(1,1), \pm(1,-1)$.

Theorem 3. The diophantine equation

$$
\begin{aligned}
e^{6}- & 27 f^{6}-g^{6}+27 h^{6}+e^{5}(3 f+4 g+2 h) \\
& +f^{5}(27 e+18 g-108 h)+g^{5}(4 e+2 f-3 h)+h^{5}(18 e-108 f-27 g) \\
& +e^{4}\left(-6 f^{2}-2 g^{2}-46 h^{2}+10 f g-8 f h-28 g h\right) \\
& +f^{4}\left(18 e^{2}+60 g^{2}+54 h^{2}+24 e g+90 e h+216 g h\right) \\
& +g^{4}\left(2 e^{2}+20 f^{2}+6 h^{2}-24 e f+10 e h-8 f h\right) \\
& +h^{4}\left(-138 e^{2}-54 f^{2}-18 g^{2}+252 e f+24 e g+90 f g\right) \\
& -17 e^{3} f^{3}-9 e^{3} g^{3}+137 e^{3} h^{3}-71 f^{3} g^{3}+243 f^{3} h^{3}+17 g^{3} h^{3} \\
& +e^{3}\left(-16 f^{2} g-34 f^{2} h+22 f g^{2}+38 f h^{2}-20 g^{2} h+55 g h^{2}\right) \\
& +f^{3}\left(-34 e^{2} g+48 e^{2} h-92 e g^{2}-270 e h^{2}-204 g^{2} h-63 g h^{2}\right) \\
& +g^{3}\left(-7 e^{2} f+30 e^{2} h+68 e f^{2}-16 e h^{2}+92 f^{2} h-34 f h^{2}\right) \\
& +h^{3}\left(-165 e^{2} f-38 e^{2} g-180 e f^{2}-34 e g^{2}-198 f^{2} g+48 f g^{2}\right) \\
& +48 e^{3} f g h-300 e f^{3} g h-100 e f g^{3} h+144 e f g h^{3} \\
& +10 e^{2} f^{2} g^{2}+204 e^{2} f^{2} h^{2}+68 e^{2} g^{2} h^{2}+30 f^{2} g^{2} h^{2}+88 e^{2} f^{2} g h \\
& -69 e^{2} f g^{2} h-236 e^{2} f g h^{2}+323 e f^{2} g^{2} h+207 e f^{2} g h^{2}-88 e f g^{2} h^{2}= \pm 1
\end{aligned}
$$


has only the solutions $(e, f, g, h) \in \mathbb{Z}$ given by

$$
(e, f, g, h)= \pm\left(e_{n}, f_{n}, g_{n}, h_{n}\right)
$$

for $n \in \mathbb{Z}$. Here the sequences $\left(e_{n}\right),\left(f_{n}\right),\left(g_{n}\right),\left(h_{n}\right)$ are binary recurrence sequences, all satisfying

$$
u_{n+2}=3 u_{n+1}+u_{n}
$$

for $u=e, f, g, h$, that are determined by their initial values for $n=0,1$, for which there are the following 27 cases:

\begin{tabular}{|rrrr|rrrr|}
\hline$e_{0}$ & $f_{0}$ & $g_{0}$ & $h_{0}$ & $e_{1}$ & $f_{1}$ & $g_{1}$ & $h_{1}$ \\
\hline \hline-5 & 1 & 3 & -1 & -2 & -3 & 0 & 1 \\
-1 & 0 & -2 & 1 & -1 & -1 & 1 & 0 \\
-1 & 1 & 2 & -1 & 2 & 1 & -1 & 0 \\
1 & 0 & 3 & -1 & 1 & 1 & 0 & 1 \\
0 & 1 & -5 & 3 & 3 & 2 & 4 & 1 \\
1 & 0 & 0 & 0 & 1 & 1 & 0 & 0 \\
0 & 0 & 1 & 0 & 0 & 0 & 1 & 1 \\
-1 & 0 & 1 & 0 & -1 & -1 & 1 & 1 \\
0 & 1 & 1 & 0 & 3 & 2 & 1 & 1 \\
2 & -1 & -1 & 1 & -1 & 0 & 2 & 1 \\
-1 & 1 & 1 & 0 & 2 & 1 & 1 & 1 \\
-1 & 0 & -1 & 0 & -1 & -1 & -1 & -1 \\
1 & -2 & -3 & 0 & -5 & -3 & -3 & -3 \\
-7 & 0 & 1 & -3 & -7 & -7 & -8 & -5 \\
\hline
\end{tabular}

\begin{tabular}{|rrrr|rrrr|}
\hline$e_{0}$ & $f_{0}$ & $g_{0}$ & $h_{0}$ & $e_{1}$ & $f_{1}$ & $g_{1}$ & $h_{1}$ \\
\hline \hline-1 & 1 & -5 & 3 & 2 & 1 & 4 & 1 \\
0 & -1 & -2 & 0 & -3 & -2 & -2 & -2 \\
2 & 0 & -1 & 1 & 2 & 2 & 2 & 1 \\
-2 & 0 & 0 & -1 & -2 & -2 & -3 & -2 \\
5 & -1 & -5 & 2 & 2 & 3 & 1 & -1 \\
1 & 0 & 0 & 1 & 1 & 1 & 3 & 2 \\
-4 & 3 & 7 & -2 & 5 & 2 & 1 & 3 \\
-1 & 0 & 1 & -1 & -1 & -1 & -2 & -1 \\
-1 & 1 & 2 & 0 & 2 & 1 & 2 & 2 \\
-3 & 0 & 1 & -2 & -3 & -3 & -5 & -3 \\
5 & -3 & -5 & 1 & -4 & -1 & -2 & -3 \\
3 & -1 & 1 & 0 & 0 & 1 & 1 & 1 \\
2 & -3 & -7 & 0 & -7 & -4 & -7 & -7 \\
\end{tabular}

Equation (2) is the most horrible diophantine equation I have ever seen completely solved.

\section{THE KEY LEMMA AND PROOFS}

3.1. The key lemma. The results of the previous section (as well as [4, Theorem 3], as was shown in [4, p. 154, bottom], and hence also the main result [4, Theorem 1] of that paper) follow from the following Key Lemma. We adopt the notation $(\xi, \varepsilon, \ldots)$ of [4]. In particular, $\xi$ is a root of $x^{3}+(9+2 \sqrt{13}) x^{2}$ $-(12+\sqrt{13}) x-\frac{11+3 \sqrt{13}}{2}=0, \sigma$ permutes the three roots of this equation, and $\varepsilon$ denotes a unit in the field $\mathbb{Q}(\xi)$. A crucial fact in the reasoning below is that $\sigma$ is a $\mathbb{Q}(\sqrt{13})$-automorphism.

The Key Lemma gives the set of solutions $\varepsilon$ of the unit equation

$$
\left(\sigma(\xi)-\sigma^{2}(\xi)\right) \varepsilon+\left(\sigma^{2}(\xi)-\xi\right) \sigma(\varepsilon)+(\xi-\sigma(\xi)) \sigma^{2}(\varepsilon)=0 .
$$

This is [4, equation (24)], and in [4] it is argued that it is equivalent to [4, equation (28)], up to multiplication by a power of $\alpha$.

Key Lemma. The unit equation (3) with $\varepsilon= \pm \theta^{a_{1}} \eta^{a_{2}} \varphi^{a_{3}} \chi^{a_{5}}$ has exactly the 27 solutions presented in the table in $[4, \mathrm{p} .155]$.

This lemma still needs a proof, since the proof presented in $[4, \S 3]$ uses the assumption that $x, y \in \mathbb{Z}$. We do this in the subsequent two sections by following a slight variant of the method of Tzanakis and de Weger $[2, \S 9]$ and using the new result on linear forms in logarithms of Baker and Wüstholz [1].

3.2. Proof of Key Lemma, part I: An upper bound. Let the index set $I=$ $\{1,2,4,5\}$, and let the matrix $U_{I}$ be defined as in $[4, \S 3.3]$. Then the row 
norm $\mathbb{N}\left[U_{I}^{-1}\right]$, defined as in $[3, \S 9]$, satisfies $\mathbb{N}\left[U_{I}^{-1}\right]<2.7352$. Let $k \in$ $\{1, \ldots, 6\}$ be such that $|\log | \varepsilon^{(k)}||$ is maximal. Then by

$$
\mathbf{b}=\mathbf{c}+U_{I}^{-1}\left(\begin{array}{l}
\log \left|\varepsilon^{(2)}\right|-\log \left|\varepsilon^{(3)}\right| \\
\log \left|\varepsilon^{(3)}\right|-\log \left|\varepsilon^{(1)}\right| \\
\log \left|\varepsilon^{(5)}\right|-\log \left|\varepsilon^{(6)}\right| \\
\log \left|\varepsilon^{(6)}\right|-\log \left|\varepsilon^{(4)}\right|
\end{array}\right)
$$

we obtain

$$
B \leq 3+2 \mathbb{N}\left[U_{I}^{-1}\right]|\log | \varepsilon^{(k)}||<3+5.4704|\log | \varepsilon^{(k)}|| .
$$

Hence, for this $k$,

$$
\text { either }\left|\varepsilon^{(k)}\right|>e^{\frac{B-3}{5.4704}} \text { or }\left|\varepsilon^{(k)}\right|<e^{-\frac{B-3}{5.4704}} .
$$

Note that

$$
N_{K / \mathbb{Q}(\sqrt{13})}(\eta)=N_{K / \mathbb{Q}(\sqrt{13})}\left(\theta^{-2} \psi\right)=N_{K / \mathbb{Q}(\sqrt{13})}(\chi)=-1, N_{K / \mathbb{Q}(\sqrt{13})}(\varphi)=1,
$$

so that

$$
\left|N_{K / \mathbb{Q}(\sqrt{13})}(\varepsilon)\right|=|\varepsilon||\sigma(\varepsilon)|\left|\sigma^{2}(\varepsilon)\right|=1 .
$$

Let $i_{0} \in\{1, \ldots, 6\}$ be such that $\left|\varepsilon^{\left(i_{0}\right)}\right|$ is minimal. We now claim that if $B \geq 100$ then

$$
\left|\varepsilon^{\left(i_{0}\right)}\right|<e^{-0.08865 B} .
$$

Indeed, assume the contrary. Then either

$$
e^{\frac{B-3}{5.4704}}<\left|\varepsilon^{(k)}\right|=\left|\sigma\left(\varepsilon^{(k)}\right)\right|^{-1}\left|\sigma^{2}\left(\varepsilon^{(k)}\right)\right|^{-1} \leq\left|\varepsilon^{\left(i_{0}\right)}\right|^{-2} \leq e^{0.1773 B},
$$

implying $B<99.7$, or

$$
e^{-\frac{B-3}{5.4704}}>\left|\varepsilon^{(k)}\right| \geq\left|\varepsilon^{\left(i_{0}\right)}\right|>e^{-0.08865 B},
$$

implying $B<5.9$.

The next step is to show that some linear form in logarithms is extremely small. Put, as in [4, p. 147],

$$
\Lambda_{i}=\log \left|\frac{\xi^{(i)}-\sigma^{2}\left(\xi^{(i)}\right)}{\xi^{(i)}-\sigma\left(\xi^{(i)}\right)} \cdot \frac{\sigma\left(\varepsilon^{(i)}\right)}{\sigma^{2}\left(\varepsilon^{(i)}\right)}\right|,
$$

which is of interest because it is related to the unit equation (3), namely by

$$
e^{\Lambda_{i}}-1=\frac{\xi-\sigma^{2}(\xi)}{\xi-\sigma(\xi)} \cdot \frac{\sigma(\varepsilon)}{\sigma^{2}(\varepsilon)}-1=-\frac{\sigma(\xi)-\sigma^{2}(\xi)}{\sigma(\xi)-\xi} \cdot \frac{\varepsilon}{\sigma^{2}(\varepsilon)} .
$$

Now notice that the unit equation (3) also leads to

$$
e^{-\Lambda_{i}}-1=\frac{\xi-\sigma(\xi)}{\xi-\sigma^{2}(\xi)} \cdot \frac{\sigma^{2}(\varepsilon)}{\sigma(\varepsilon)}-1=-\frac{\sigma^{2}(\xi)-\sigma(\xi)}{\sigma^{2}(\xi)-\xi} \cdot \frac{\varepsilon}{\sigma(\varepsilon)} .
$$

For $i=i_{0}$ we have

$$
\max \left\{\left|\sigma\left(\varepsilon^{\left(i_{0}\right)}\right)\right|,\left|\sigma^{2}\left(\varepsilon^{\left(i_{0}\right)}\right)\right|\right\} \geq\left|\sigma\left(\varepsilon^{\left(i_{0}\right)}\right)\right|^{1 / 2}\left|\sigma^{2}\left(\varepsilon^{\left(i_{0}\right)}\right)\right|^{1 / 2}=\left|\varepsilon^{\left(i_{0}\right)}\right|^{-1 / 2},
$$

hence

$$
\min \left\{\left|e^{\Lambda_{i_{0}}}-1\right|,\left|e^{-\Lambda_{i_{0}}}-1\right|\right\}<10.117\left|\varepsilon^{\left(i_{0}\right)}\right|^{3 / 2}<10.117 e^{-0.132975 B} .
$$


By $B \geq 100$ this implies

$$
\left|\Lambda_{i_{0}}\right|<10.118 e^{-0.132975 B},
$$

an inequality which replaces [4, equation (34)].

As explained in $[4, \S 3.2]$, we may assume that $i_{0}=1$. We find (see [1] for the definition of the heights $\left.h, h^{\prime}\right)$ :

\begin{tabular}{|c|cccc|}
\hline$\cdot$ & $\eta$ & $\varphi$ & $\theta^{-2} \psi$ & $\chi$ \\
\hline \hline$h(\cdot)<$ & 2.5302 & 1.9942 & 3.4366 & 2.5302 \\
$h^{\prime}(\cdot)<$ & 0.4217 & 0.3324 & 0.5728 & 0.4217 \\
\hline
\end{tabular}

From the main theorem of Baker and Wüstholz [1], with $n=4, d=6$, we infer

$$
\left|\Lambda_{1}\right|>e^{-1.4524 \times 10^{19} \log B} .
$$

Now (4) and (5) imply

$$
B<5.4670 \times 10^{21},
$$

which is slightly worse than the (incorrect) [4, equation (36)], and exactly equal to the bound given in the Correction to [4].

3.3. Proof of Key Lemma, part II: Reducing the upper bound. Using (4) and (6), we follow the line of argument as presented in [4, §3.4]. Note that in the present situation, [2, Proposition 3.1] can be stated as

$$
\text { if } l>\sqrt{19} B_{0} \text { then } B \leq\left\lfloor\frac{1}{0.132975} \log \frac{10.118 C}{\sqrt{l^{2}-3 B_{0}^{2}}-4 B_{0}}\right\rfloor,
$$

where $C$ is a parameter defining the lattice, $B_{0}$ is an upper bound for $B$, and $l=\min |x|$, the minimum taken over all nonzero lattice points $x$. Then certainly $l \geq\left|b_{1}\right| / 2 \sqrt{2}$.

Our new upper bound $B_{0}$ is only a little bit larger than the old incorrect one used in $[4, \S 3.4]$. Fortunately, this means that in the first reduction step it is sufficient to take again $C=10^{96}$. Hence again $\left|b_{1}\right|>5.1249 \times 10^{23}>$ $2 \sqrt{2} \sqrt{19} 5.4670 \times 10^{21}$, and now (7) implies $B \leq 1277$. That this is not as good as the result of the first reduction step of [4] is due to the fact that (4) is weaker than [4, equation (34)]. This is the price we have to pay for removing the assumption $x, y \in \mathbb{Z}$.

For the second reduction step we take $C=10^{17}$, and we take a somewhat closer look at the lattice. Let $\mathscr{B}$ be the matrix containing as columns the output of the $L^{3}$-algorithm, i.e., the vectors $b_{1}, b_{2}, b_{3}, b_{4}$. We computed

$$
\mathscr{B}=\left(\begin{array}{rrrr}
4617 & 2580 & -5838 & 9956 \\
-5307 & -2829 & -3883 & 12592 \\
-1864 & 357 & -16173 & -8467 \\
-2696 & 9770 & 2169 & 4283
\end{array}\right) \text {, }
$$


with $\left|b_{1}\right|>7760.4$. Now $7760.4<2 \sqrt{2} \sqrt{19} 1277$, so we cannot apply (7) directly with $l$ replaced by $\left|b_{1}\right| / 2 \sqrt{2}$. However, it is not difficult to compute $l$. Namely, for every $x=\left(x_{1}, x_{2}, x_{3}, x_{4}\right)^{t}$ in the lattice there is a $y=\left(y_{1}, y_{2}, y_{3}, y_{4}\right)^{t} \in \mathbb{Z}^{4}$ such that $x=\mathscr{B} y$. Put $\mathscr{B}^{-1}=\left(b_{i, j}\right)$. Then if $|x|<7760.5$ it follows that $\left|y_{i}\right|<7760.5 \sum_{j=1}^{4}\left|b_{i, j}\right|$, which is easily seen to imply $\left|y_{1}\right| \leq 1,\left|y_{2}\right| \leq 1, y_{3}=y_{4}=0$. Hence it is easy to find the nonzero lattice point nearest to the origin, which in fact is $b_{1}$. Thus in (7) we may take $l=\left|b_{1}\right|>7760.4$, and this leads to $B \leq 253$.

We did a third reduction step with $C=10^{14}$. Then the output of the $L^{3}$ algorithm are the column vectors $b_{1}, b_{2}, b_{3}, b_{4}$ of the matrix

$$
\mathscr{B}=\left(\begin{array}{rrrr}
-966 & 772 & 737 & 2968 \\
-1439 & 1121 & -33 & -2193 \\
-43 & 984 & 1637 & -1525 \\
591 & 1343 & -496 & 2063
\end{array}\right),
$$

so that $\left|b_{1}\right|>1831.6$. As in the second reduction step above, we found from $\left|y_{i}\right|<1832 \sum_{j=1}^{4}\left|b_{i, j}\right|$ that $\left|y_{1}\right| \leq 2,\left|y_{2}\right| \leq 1,\left|y_{3}\right| \leq 2, y_{4}=0$. Hence it is easy to show that the nonzero lattice point nearest to the origin is $b_{1}$. Thus in (7) we may take $l=\left|b_{1}\right|>1831.6$, and this leads to $B \leq 209$.

For $100 \leq B \leq 210$ we checked (4) in 18-digit precision, which is amply sufficient. Note that by $\left|\Lambda_{1}\right| /|\log | \xi^{(1)}||<0.5$, the value of $b_{5} \in \mathbb{Z}$ is determined uniquely by $b_{2}, b_{3}, b_{4}$. Hence there are $(2 \times 210+1)^{2}-(2 \times 99)^{2} \approx 6.7 \times 10^{7}$ possibilities to be checked. There are 31 solutions, which we further checked for [4, equation (28)]. None survived.

For $50 \leq B \leq 100$ we can replace (4) by the somewhat weaker inequality

$$
\left|\Lambda_{i_{0}}\right|<10.199 e^{-0.128874 B} .
$$

We tested this inequality for all $\approx 7.2 \times 10^{6}$ possibilities, and found 20272 solutions, none of which satisfies [4, equation (28)].

Finally we checked $[4$, equation $(28)]$ directly for all $\approx 10^{8}$ possibilities with $B \leq 50$, and this produced exactly the 27 solutions already known. This completes the proof of the lemma.

Total computation time was less than 2 hours on a 80486 personal computer.

\subsection{Proofs of theorems.}

Proof of Theorem 1. Equation (1) leads to (and is in fact equivalent to)

$$
x-y \xi=\varepsilon .
$$

The unit equation (3) is obtained by applying the $\mathbb{Q}(\sqrt{13})$-automorphisms $\sigma, \sigma^{2}$ of $\mathbb{Q}(\xi)$ to equation (8), and then eliminating $x, y$ from the three conjugate equations so obtained. This argument remains valid if we take $x, y \in \mathbb{Q}(\sqrt{13})$ such that $x-y \xi \in \mathscr{O}$. So does [4, equation (25)], which can be used to recover $x$ and $y$ from a solution $\varepsilon$ of the unit equation (3). (Note that [4, equation (26)] uses $x, y \in \mathbb{Z}$, so we cannot use it here).

To produce an algebraic integer $x-y \xi$ in equation (8), it is not necessary for $x, y$ to be algebraic integers. The reason is that $\zeta=(3+\xi) / \sqrt{13}$ is an algebraic integer too. Hence for the moment, for the solutions of (1) we take $x, y \in \frac{1}{\sqrt{13}} \mathscr{O}$ such that $x+3 y \in \mathscr{O}$. 
From the Key Lemma, by [4, equation (25)], we computed the 27 classes of solutions of equation (1). It is easily seen (by working modulo 13) that 25 of the 27 classes do not lead to any solution $x, y \in \mathscr{O}$ at all. The remaining two classes are given by $\varepsilon= \pm \alpha^{m}$ and $\varepsilon= \pm \alpha^{m} \theta^{8} \eta \varphi^{2} \chi^{4}$, and they lead to only $x, y \in \mathscr{O}$, namely exactly those mentioned in the statement of the theorem. This completes the proof of Theorem 1 .

Proof of Theorem 2. Using an argument similar to [4, equation (25), (26)], we found that the only solutions of (1) with $x, y \in \frac{1}{\sqrt{13}} \mathbb{Z}$ such that $x+3 y$ is an algebraic integer are $(x, y, n)=\left(\frac{-4}{\sqrt{13}}, \frac{-3}{\sqrt{13}},-1\right),\left(\frac{-3}{\sqrt{13}}, \frac{1}{\sqrt{13}}, 1\right)$. On putting $v=x \sqrt{13}, w=y \sqrt{13}$ and taking the $\mathbb{Q}(\sqrt{13})$-norm of $(1)$, we obtain Theorem 2(i). Further, using a similar argument with $x, y$ replaced by $t=x+3 y, u=$ $y \sqrt{13}$ and requiring $t, u \in \mathbb{Z}$, we obtain Theorem 2(ii). We leave the details of deriving Theorem 2 from the Key Lemma to the reader.

Proof of Theorem 3. In view of the fact that $\zeta=(3+\xi) / \sqrt{13} \in \mathscr{O}$, we write

$$
x-y \xi=(x+3 y)-y \sqrt{13} \zeta=\left(e+f \frac{1+\sqrt{13}}{2}\right)-\left(g+h \frac{1+\sqrt{13}}{2}\right) \zeta,
$$

where $e, f, g, h \in \mathbb{Z}$. We substitute this in equation (1) and then take the $\mathbb{Q}(\sqrt{13})$-norm. The Key Lemma immediately yields the result (in fact, is equivalent to it), where again we leave further details to the reader.

\section{BIBLIOGRAPHY}

1. A. Baker and G. Wüstholz, Logarithmic forms and group varieties, J. Reine Angew. Math. 442 (1993), 19-62.

2. N. Tzanakis and B. M. M. de Weger, On the practical solution of the Thue equation, J. Number Theory 31 (1989), 99-132.

3. $\ldots$, How to explicitly solve a Thue-Mahler equation, Compositio Math. 84 (1992), 223288.

4. B. M. M. de Weger, A hyperelliptic diophantine equation related to imaginary quadratic number fields with class number 2, J. Reine Angew. Math. 427 (1992), 137-156; Correction: 441 (1993), 217-218.

Econometric Institute, Erasmus University Rotterdam, P.O. Box 1738, 3000 DR RotTERDAM, THE NETHERLANDS

E-mail address: dweger@is.few.eur.nl 